\title{
Programas de intervenções comportamentais e de desenvolvimento intensivas precoces para crianças com TEA: uma revisão de literatura
}

Early intensive behavioral and developmental intervention programs for children with ASD: a literature review

Programas de intervenciones comportamentales y de desarrollo intensivas precoces para niños con TEA: una revisión de literatura

\section{Ana Carolina Wolff Mota}

Doutoranda na Universidade Federal de Santa Catarina, Florianópolis, Santa Catarina, Brasil. anacwolff@gmail.com

ORCID - https://orcid.org/0000-0003-3767-1310

\section{Mauro Luis Vieira}

Professor doutor na Universidade Federal de Santa Catarina, Florianópolis, Santa Catarina, Brasil. maurolvieira@gmail.com

ORCID - https://orcid.org/0000-0003-0541-4133

Adriano Henrique Nuernberg

Doutor pela Universidade Federal de Santa Catarina, Florianópolis, Santa Catarina, Brasil.

adrianoh@outlook.com

ORCID - https://orcid.org/0000-0002-7875-8490

Recebido em 27 de agosto de 2019

Aprovado em 11 de março de 2020

Publicado em 10 de abril de 2020

\section{RESUMO}

Indivíduos diagnosticados com Transtorno do Espectro do Autismo (TEA) apresentam comprometimento qualitativo para interagir socialmente de forma recíproca, para discriminar o sentido social das experiências com outros e, por conseguinte, prejuízo significativo no processo de desenvolvimento de habilidades sociocomunicativas. Sinais de autismo podem ser identificados desde o primeiro ano de vida, o que representa possibilidade de iniciar intervenção precoce - sendo esta um importante preditor na recuperação funcional desse transtorno do neurodesenvolvimento. O objetivo do presente estudo é revisar, na literatura científica, procedimentos de intervenção comportamentais e de desenvolvimento intensivas e precoce, em crianças diagnosticadas com TEA, que revelem evidência de resultados. Foram pesquisadas cinco bases de dados internacionais, nas áreas de saúde e educação, como fontes da busca, quais sejam: a) Web of Science; b) Scopus; c) Psyclnfo; d) Eric; e, e) BVS. A partir disso, foram selecionados 95 artigos a partir dos critérios de inclusão adotados e identificou-se que as principais áreas de desenvolvimento-alvos das intervenções são habilidades de comunicação, interação social e cognição; o tempo médio pode variar, contudo, a recomendação é de que haja intensidade de, no mínimo, 3 horas por dia; os contextos de intervenção variam e envolvem predominantemente ambientes clínicos ou domésticos, cujos agentes de intervenção são técnicos e pais. Quanto aos ingredientes ativos nos efeitos das intervenções, quanto mais 
http://dx.doi.org/10.5902/1984686X41167

nova a criança e mais alto for o QI, maior a probabilidade de desfechos favoráveis na intervenção; em relação aos principais indicadores de qualidade, os programas são avaliados a partir de ganhos nas habilidades cognitivas, de linguagem, adaptativas e sociais. Tais resultados podem ser convertidas em diretrizes na implementação de programas de intervenção precoce para crianças com autismo.

Palavras-chave: Autismo; intervenções intensivas precoces no desenvolvimento; programas de intervenção; evidência.

\section{ABSTRACT}

Individuals diagnosed with Autism Spectrum Disorder (ASD) have a qualitative commitment to interact socially in a reciprocal manner, to discriminate the social meaning of experiences with others; therefore, significantly impairing the process of developing socio-communicative skills. Signs of autism can be identified from the first year of life, which represents the possibility of initiating early intervention - an important predictor in the functional recovery of this neurodevelopmental disorder. The purpose of this study is to review, in the scientific literature, early intensive behavioral and developmental intervention procedures in children diagnosed with ASD, which can reveal evidence of results. Five international databases were searched as sources, in the areas of health and education, namely: a) Web of Science, b) Scopus, c) Psyclnfo, d) Eric, e) VHL. Next, the selection of 95 articles was based on the adopted inclusion criteria and led to the identification that key developmental areas of interventions are communication skills, social interaction and cognition; that average time can vary, however, an intensity of at least 3 hours per day is recommended; contexts of intervention vary and involve predominantly clinical or residential environments, whose intervention agents are technicians and parents. As for the active components in the effects of the interventions, the younger the child and the higher the IQ, the greater the probability of favorable outcomes in the intervention; regarding the key quality indicators, the programs are evaluated based on gains in cognitive, language, adaptive and social skills. Such results can be turned into guidelines in the implementation of early intervention programs for children with autism.

Keywords: Autism; early intensive interventions in development; intervention programs; evidence.

\section{RESUMEN}

Individuos diagnosticados con Trastorno del Espectro de Autismo (TEA) presentan una dificultad cualitativa para interactuar socialmente de forma recíproca, para distinguir el sentido social de las experiencias con otros y, por consiguiente, perjuicio significativo en el proceso de desarrollo de habilidades sociocomunicativas. Las señales de autismo pueden ser identificadas desde el primer año de vida, lo que representa una posibilidad de iniciar una intervención precoz siendo este un importante previsor en la recuperación funcional de este trastorno del neurodesarrollo. El objetivo del presente estudio es revisar, en la literatura científica, procedimientos de intervención comportamentales y de desarrollo intensivas y precoz, en niños diagnosticados con TEA, que revelen evidencia de resultados. Se han investigado cinco bases de datos internacionales, en las áreas de salud y educación, como fuentes de consulta, tales como: a) Web of Science, b) Scopus, c) Psyclnfo, d) Eric, e) BVS. De ahí, se seleccionaron 95 artículos a partir de los criterios de inclusión adoptados y se identificó que las principales áreas de desarrollos objetivos de las intervenciones son habilidades de comunicación, interacción social y cognición; el tiempo promedio puede variar, sin embargo, la recomendación es de que haya intensidad de, por lo menos, 3 horas al día; los contextos de intervención varían e involucran predominantemente ambientes 
clínicos o domésticos, cuyos agentes de intervención son técnicos y padres. Con relación a los ingredientes activos en los efectos de las intervenciones, cuanto más joven es el niño y más alto es el QI, mayor es la probabilidad de resultados favorables en la intervención; en relación a los principales indicadores de calidad, los programas son evaluados a partir de beneficios en las habilidades cognitivas, de lenguaje, adaptativas y sociales. Tales resultados pueden ser convertidos en directrices en la implementación de programas de intervención precoz para niños con autismo.

Palabras clave: Autismo; intervenciones intensivas precoces en el desarrollo; programas de intervención; evidencia.

\section{Introdução}

O autismo é classificado como transtorno do neurodesenvolvimento e é reconhecido sumariamente pela presença de prejuízos significativos em habilidades sociocomunicativas e, além disso, por essas pessoas apresentarem comportamentos e interesses estereotipados, de diversas ordens. Com os avanços em técnicas de rastreamento e diagnóstico cada vez mais precoces, a intervenção também passou a ser possível mais precocemente - ainda que, na prática, as famílias venham tendo acesso ao diagnóstico dos filhos tardiamente (ZANON; BACKES; BOSA, 2017).

A intervenção precoce é fator fundamental para a melhora do quadro clínico do autismo ao longo do processo de desenvolvimento, embora ainda não se tenham fortes fundamentos de pesquisa que incluam investigações sobre o valor a longo prazo das intervenções precoces (TONGE et al., 2014). Os ganhos infantis nas habilidades sociocomunicativas e de desenvolvimento são observados após a intervenção precoce, de modo que estudos revisados destacaram sua viabilidade, fornecendo evidências preliminares de que a intervenção para bebês em risco pode ser benéfica para os bebês e para os pais (BRADSHAW et al., 2015).

Pessoas com autismo apresentam, desde antes dos três anos de idade, comprometimento qualitativo para interagir socialmente de forma recíproca, para discriminar a função social das experiências com outros e, por conseguinte, prejuízo significativo no processo de desenvolvimento de habilidades sociocomunicativas. Tendem a ter dificuldades para o desenvolvimento da capacidade de imaginar, o que implica em uma tendência a adotar padrões restritos e repetitivos de atividades e interesses, como adoção de padrões motores, adesão insistente e rígida a rotinas e, ainda, alterações nas respostas sensoriais (com hiper ou hiporresponsividade) (Associação Americana de Psiquiatria - APA, 2014). 
http://dx.doi.org/10.5902/1984686X41167

Sinais de alerta para o autismo são passíveis de identificação já nos primeiros meses de vida do bebê, embora não pareçam ser sinais específicos de transtorno do espectro do autismo, como alterações de sono e comportamento alimentar (BOSA; ZANON, 2016). Estes sinais também não diferenciam as crianças que apresentam atraso no desenvolvimento daquelas com autismo. Após os 6 meses de idade, sim, comportamentos podem evidenciar mais o autismo, de modo que tende a haver um declínio da frequência de comportamentos como olhar para faces, sorriso social e vocalizações (OZONOFF et al., 2010). A presença de comportamentos indicativos do autismo fica mais marcante na medida que em avança o processo de desenvolvimento ontogenético - desvelando, então, as lacunas biológicas do desenvolvimento que interferem nas capacidades de engajamento social, habilidades sociocomunicativas, entre outros.

As crianças com autismo, então, embora cresçam entre artefatos culturais que foram acumulados ao longo da história de seu grupo cultural, têm comprometida a capacidade de se aprender espontaneamente, nessas interações, aquilo que a humanidade acumulou - a linguagem, as convenções sociais, o uso de ferramentas simbólicas em geral. Por razões biológicas, não possuem a "chave sociocognitiva" que a liga a todo esse arsenal sociocultural (TOMASELLO, 2003). Os indivíduos mais gravemente afetados pelo autismo são incapazes de compreender as outras pessoas como agentes intencionais (agentes mentais) iguais a eles próprios (TOMASELLO, 2003). A falta dessa habilidade pode explicar, assim, a profunda dificuldade que pessoas com autismo têm para se envolver em habilidades de aprendizagem cultural típicas da espécie - já que os comportamentos de imitação se apresentam também falhos.

Crianças com autismo podem compreender certos aspectos da ação intencional e, mesmo que desenvolvam alguma habilidade de aprendizagem social, não se tem evidências de que possam ser capazes de engajamentos socioafetivos de qualidade idêntica a crianças de desenvolvimento típico. Há evidências que sugerem que crianças com autismo parecem ter uma falta de motivação para compartilhar intencionalidade com seus co-específicos, limitando a criança de atuação conjunta para a criação cultural com outros indivíduos. Um exemplo é a percepção de estar sendo imitado, que é comprometido em crianças com autismo (CONTALDO et al., 2016). Outras evidências sugerem, ainda, que as pessoas com autismo, embora tenham capacidade para compartilhar intencionalidade, têm déficit cognitivo devido à ausência de habilidades perceptuais que as 
http://dx.doi.org/10.5902/1984686X41167

permitam compreender estados psicológicos de si e de seus co-específicos em contextos sociais complexos (TOMASELLO, 2008).

Como núcleo da habilidade de atenção compartilhada, a intencionalidade no comportamento da criança pode ser expressa primeiramente por meio de gestos como apontar, mostrar, dar objetos, alternando seu olhar entre os objetos e a face do parceiro social. Entretanto, comportamentos protoimperativos devem ser criteriosamente diferenciados dos comportamentos protodeclarativos: os primeiros visam à busca de assistência (por ex., apontar para algo para pedir) enquanto os segundos revelam a intenção de compartilhar um interesse (por ex., apontar para algo com intenção explícita de mostrar para o parceiro, querendo interagir). Criança com autismo podem apresentar o primeiro tipo de comportamento social (cuja leitura de intenção do seu desejo de acessar algo cabe ser interpretado pelo parceiro), mas apresentam menos frequentemente 0 segundo tipo (CANCINO, 2013).

O comprometimento da habilidade de atenção compartilhada tem sido referida com um importante marcador preditivo para identificação precoce de TEA (BOSA; ZANON, 2016; MARQUES; BOSA, 2015). Os comportamentos de atenção compartilhada podem, contudo, mudar de forma e função, de modo que requer uma essencial diferenciação, por vezes sutis aos olhos de um observador clínico pouco treinado. Os comportamentos podem ser por resposta ou por iniciativa de interação social com objetivo de compartilhar atenção (ZANON; BACKES; BOSA, 2015).

O comportamento de Resposta de Atenção Compartilhada (RAC) é caracterizado pela habilidade de seguir a direção do olhar, orientação facial e/ou gesto de apontar de outra pessoa em direção a um objeto. Esses comportamentos implicam em desenvolvimento precoce de uma consciência de que outras pessoas têm estados mentais e podem ser indicados por sinais sociais. Já a habilidade de Iniciativa de Atenção Compartilhada (IAC) é uma habilidade mais avançada, porque se caracteriza pela habilidade de utilizar o contato ocular e/ou gestos (como apontar, mostrar), com finalidade de iniciar espontaneamente um contexto de atenção compartilhada (BOSA; ZANON, 2016).

Os construtos de RAC e IAC precisam ser discutidos no caso do autismo porque os primeiros costumam ser não só mais comuns que os segundos, como os segundos podem ser facilmente confundidos com emulação. Essa diferenciação é particularmente importante no caso da formulação de instrumentos de avaliação. Itens que avaliam atenção compartilhada podem não se ocupar de tal diferenciação, fragilizando o processo avaliativo, ao permitir que 
http://dx.doi.org/10.5902/1984686X41167

uma aprendizagem por emulação (em tese mais preservada em criança com autismo) possa ser capturada no mesmo nível de habilidade que a IAC ou RAC (MARQUES; BOSA, 2015).

A fase inicial do desenvolvimento humano merece especial atenção, porque imperam os aspectos não-verbais, não-linguísticos do desenvolvimento humano - que, embora não sejam os únicos que afetam as pessoas com autismo, trazem as chaves para explicar a condição central do transtorno: a falha na capacidade de compreender a intencionalidade. Não por acaso pessoas com autismo são tão literais: se falham na possibilidade de compreender claramente a intenção do outro, como entender segundas intenções? (TOMASELLO et al., 2005)

A natureza difusa e refratária do autismo e os avanços recentes no tratamento psicológico e educacional de crianças com TEA têm tornado cada vez mais urgente que as crianças sejam diagnosticadas o mais cedo possível. Assim, com o diagnóstico precoce, crianças podem ser encaminhadas para serviços de intervenção adequados e eficazes, visto que a intervenção precoce tem sido um importante preditor da recuperação funcional desse transtorno do neurodesenvolvimento (SÁNCHEZ-RAYA et al., 2015). Há um corpo bem estabelecido de evidências empíricas que demonstram eficácia da intervenção precoce para crianças com autismo, apontando melhorias principalmente na promoção do desenvolvimento de habilidades, melhora de comportamentos da criança e redução do estresse experimentados pela criança e pela família (TONGE et al., 2014). Quando se refere à intervenção precoce, os programas precisam ser analisados com maior critério, pois na maior parte não se define a faixa etária específica. O termo "precoce" pode ser compreendido como a primeira infância em termos amplos (até o final da idade pré-escolar - 6 anos) (LAMPREIA, 2007; TONGE et al., 2014), ou pode, ainda, se referir a fases mais iniciais, anterior aos 4 anos de idade (ROGERS; DAWSON, 2014). No presente estudo, foram contemplados estudos que tratam, genericamente, da primeira infância (crianças menores de 60 meses).

Seja qual for o programa, tem sido cada vez mais relevante que as intervenções com crianças com autismo sigam os princípios de práticas baseadas em evidências, integrando as melhores evidências de pesquisa com a experiência clínica profissional e os valores e preferências dos pacientes. Essas práticas envolvem o aprendizado do profissional que, ao cuidar do paciente, cria a necessidade de informações sobre seus problemas. Tem na literatura de pesquisa fonte essencial de consulta e reconhece que evidências de melhores práticas de hoje podem modificar num futuro próximo. Em razão da importância de manter-se atualizado, é necessário incorporar ferramentas das práticas baseadas em evidências, rastreando e avaliando criticamente resultados de pesquisas para incorporar à prática clínica diária 
http://dx.doi.org/10.5902/1984686X41167

(AKOBENG, 2005).Embora não haja consenso sobre que modelo de intervenção é mais adequado para crianças com TEA, reconhece-se que os melhores meios de tratamento sejam os enfoques psicoeducativos (que visam ao desenvolvimento de habilidades por meio de estratégias de ensino) e com apoio comunitário (acolhendo e instrumentalizando família e comunidade para a interação com a pessoa com autismo) (MARTOS-PÉREZ; LLORENTECOMÍ, 2013). Nesse sentido, existem significativos acordos em relação aos princípios metodológicos gerais que devem guiar qualquer programa e atuação terapêutica e educativa com pessoas com autismo. Os alvos básicos de qualquer tratamento para crianças com TEA são: 1) estimulação do desenvolvimento social e comunicativo; 2) aprimoramento do aprendizado e capacidade de solucionar problemas; 3) redução de comportamentos disfuncionais, que interferem no aprendizado e no acesso às oportunidades de experiências no cotidiano; 4) auxílio às famílias para lidarem com o autismo (BOSA, 2006).

Os programas podem ser classificados de formas diferentes. A mais usual classificação divide os programas em três tipos: 1) práticas baseadas em intervenções; 2) modelos globais de tratamento (modelos de tratamento compreensivo); 3) estudos psicofarmacológicos. Nos programas do primeiro tipo (práticas baseadas em intervenções), são utilizados de procedimentos de aprendizagem ou práticas específicas para potencializar o desenvolvimento e aprendizagem de condutas específicas ou ainda para reduzir comportamentos problemas num tempo relativamente breve (SALVADÓ-SALVADÓ et al., 2012). Essas práticas podem ser partes de programas maiores, mais bem estruturados, como os de segundo tipo, que incluem os modelos globais de tratamento, que têm um marco teórico especificado, com organização conceitual e são formados por multicomponentes que se integram. Entre eles, estão o TEACCH (Treatment and Education of Autistic and Related Communication Handicapped Children), o Modelo Denver ou o Programa SCERTS (Programa educacional cujo nome é acróstico das áreas de desenvolvimento abrangidas: Social Comunication, Emotional Regulation \& Transactional Support). Já o terceiro tipo de programas reúne as propostas de intervenções biomédicas, derivadas de estudos psicofarmacológicos, além de intervenções de restrições alimentares, como glúten e caseína (SALVADÓ-SALVADÓ et al., 2012).

Dentre os modelos globais de tratamento, programas de intervenção precoce abrangentes comportamentais (baseados na psicologia comportamental) e desenvolvimentistas (baseados na psicologia do desenvolvimento) têm apontado evidências de eficácia na evolução das crianças com autismo. Um estudo com 78 crianças com TEA menores de 3 anos receberam intervenções, comportamentais ou ecléticas, ao longo de um 
http://dx.doi.org/10.5902/1984686X41167

ano, e obtiveram melhoras significativas na capacidade verbal e redução da gravidade dos sintomas do autismo. Verificou-se também que há diferentes respostas às intervenções: melhores resultados de habilidades adaptativas foram observados quanto mais avançada a idade da mãe também em crianças com maior gravidade nos sintomas do autismo, porém, com melhor capacidade verbal (BEN-ITZCHAK; ZACHOR, 2011). Num estudo comparativo de abordagens de tratamento, a intervenção analítica comportamental intensiva (relação adulto: criança 1: 1, 25-40h/ semana) promoveu maiores escores em todos os domínios de habilidades, quando comparada com os resultados de dois grupos - um que recebeu intervenções eclética intensiva em sala de aula de educação especial pública (combinação de métodos, proporção 1:1 ou 1:2, com 30h/semana) e um que recebeu intervenção precoce não intensiva em programa público (combinando métodos, em pequenos grupos, por 15h/semana). Verificou-se taxas de aprendizado substancialmente mais altas para as crianças do primeiro grupo, em todos os domínios - exceto nas habilidades motoras que as diferenças estatísticas não foram significativas (HOWARD et al., 2005).

A despeito da recomendação de intervenções intensivas, quando se trata de intervenção precoce, nem sempre é uma possibilidade, principalmente em razão de recursos econômicos. Um estudo na Índia, com 52 crianças com TEA (leve a grave) submetidas a intervenção domiciliar de baixa intensidade, apontou melhoras estatística e clínicas na gravidade do autismo, com aquisição de habilidades sociais e de linguagem. Tal modelo de intervenção se mostrou eficaz, sendo indicados principalmente para ambientes de cuidados primários $\mathrm{e}$ comunitários (NAIR et al., 2014). Outro estudo, nos EUA, com crianças com TEA que receberam intervenção com um programa TEACCH na modalidade domiciliar e de baixa intensidade, demonstrou que há benefícios para as crianças, ao reduzir os sintomas autistas e comportamentos mal-adaptativos, bem como há benefícios para os pais, com a diminuição do estresse, indicando que o envolvimento destes no programa de tratamento do filho é um fator crucial e contribuir para a eficácia do tratamento (D'ELIA et al., 2014).O presente estudo tem como objetivo geral revisar na literatura evidências científicas relativas a intervenções comportamentais e de desenvolvimento, intensivas e precoces, para crianças com TEA. Os objetivos específicos estão em termos de identificar e caracterizar: a) áreas de desenvolvimento mais afetadas pelas intervenções; b) o tempo médio de intervenção sistematizada que as crianças recebem; c) os principais contextos e agentes das intervenções; d) os ingredientes ativos nos efeitos das intervenções; e) os principais indicadores de qualidade dos programas. 
http://dx.doi.org/10.5902/1984686X41167

\section{Método}

A presente revisão sistemática da literatura, revisou estudos que apontassem evidências científicas de eficácia sobre intervenções comportamentais e de desenvolvimento, intensivas e precoces, para criança com TEA, por meio de métodos de busca sistemática e estruturada de estudos em bases de dados científicas de saúde e educação. Os objetivos específicos orientaram a sistematização de informações extraídas dos textos pesquisados, possibilitando, assim, suporte para futura tomada de decisão e melhorias na prática clínica (que são objetos da tese de doutorado de um dos autores). Este estudo compreendeu três etapas, cujo fluxograma da busca eletrônica conduzida no trabalho está apresentado na Figura 1.

Figura 1 - Etapas do estudo 1

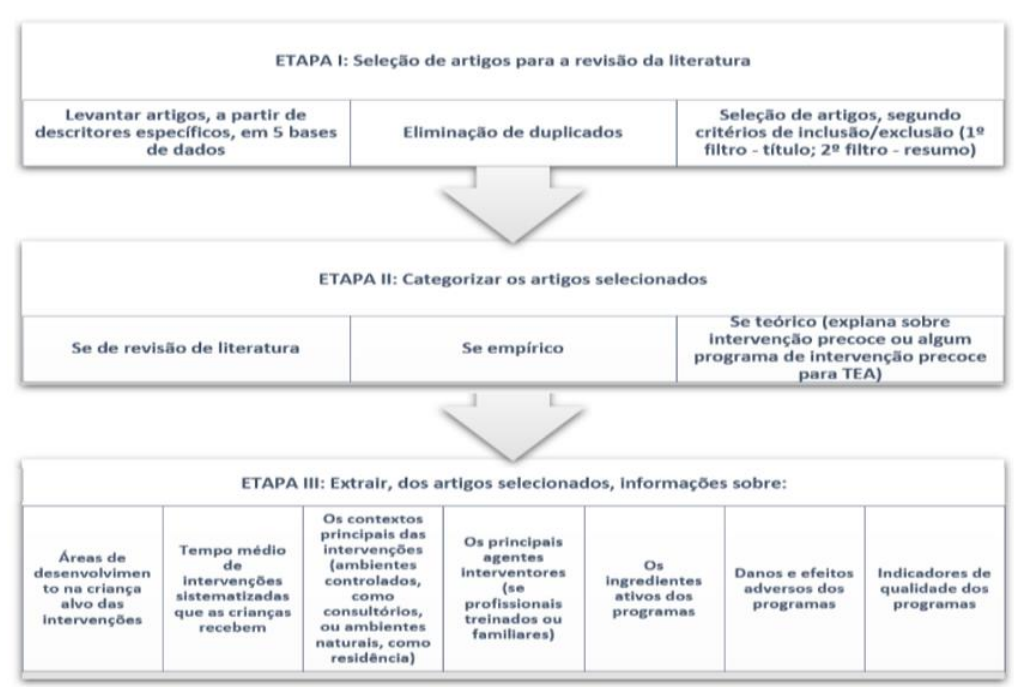

Fonte: elaborado pelos autores (2018).

A etapa 1 envolveu um levantamento na literatura de artigos que tratam de resultados favoráveis da intervenção precoce no desenvolvimento de crianças com TEA, seja por meio de estudos empíricos, teóricos ou de revisão de literatura). Foram pesquisadas cinco bases de dados internacionais, nas áreas de saúde e educação, como fontes da busca, quais sejam: a) Web of Science, b) Scopus, c) Psyclnfo, d) Eric, e) BVS. Estas bases foram escolhidas em função da sua relevância científica mundial nas áreas de saúde e educação, em geral, e na área da Psicologia - áreas de conhecimento pelas quais perpassam o tema do presente estudo (intervenção psicológica sobre o desenvolvimento de crianças com autismo). 
http://dx.doi.org/10.5902/1984686X41167

Para pesquisar em cada uma das bases de dados on-line, realizou-se o acesso a partir do Acesso CAFE - Comunidade Acadêmica Federada, do Portal de Periódicos CAPES. Os descritores foram: Autism* AND "early intervention*". Em cada base de dados, adotou-se filtro de tempo (de 2008-2017), de idioma (inglês, espanhol e português) e tipo de documento (artigos). As buscas foram realizadas ao longo do mês de junho de 2018.

Como critérios de inclusão, priorizou-se artigos que apresentassem revisões de literatura sobre intervenção precoce e autismo, estudos empíricos com mais de 10 participantes e artigos que tratavam de programas de intervenção abrangentes comportamentais e desenvolvimentistas. Foram excluídos os artigos que não atendiam a esses critérios.

\section{Resultados e discussão}

Da soma dos 2991 artigos reunidos a partir das cinco bases de dados ${ }^{1}$ na primeira etapa, restaram 95, após eliminação de duplicados (1281), daqueles que não atenderam aos critérios de inclusão pelas informações dos títulos (1555) e pelas informações dos resumos (60). Dos 95 artigos contemplados nessa revisão, 32 são de revisão de literatura, 46 empíricos e 17 teóricos. O processo realizado, ao longo do mês de junho de 2018, está representado no Fluxograma do processo de seleção dos artigos incluídos neste estudo (Figura 2).

Figura 2 - Fluxograma do processo de seleção dos artigos incluídos no estudo 1

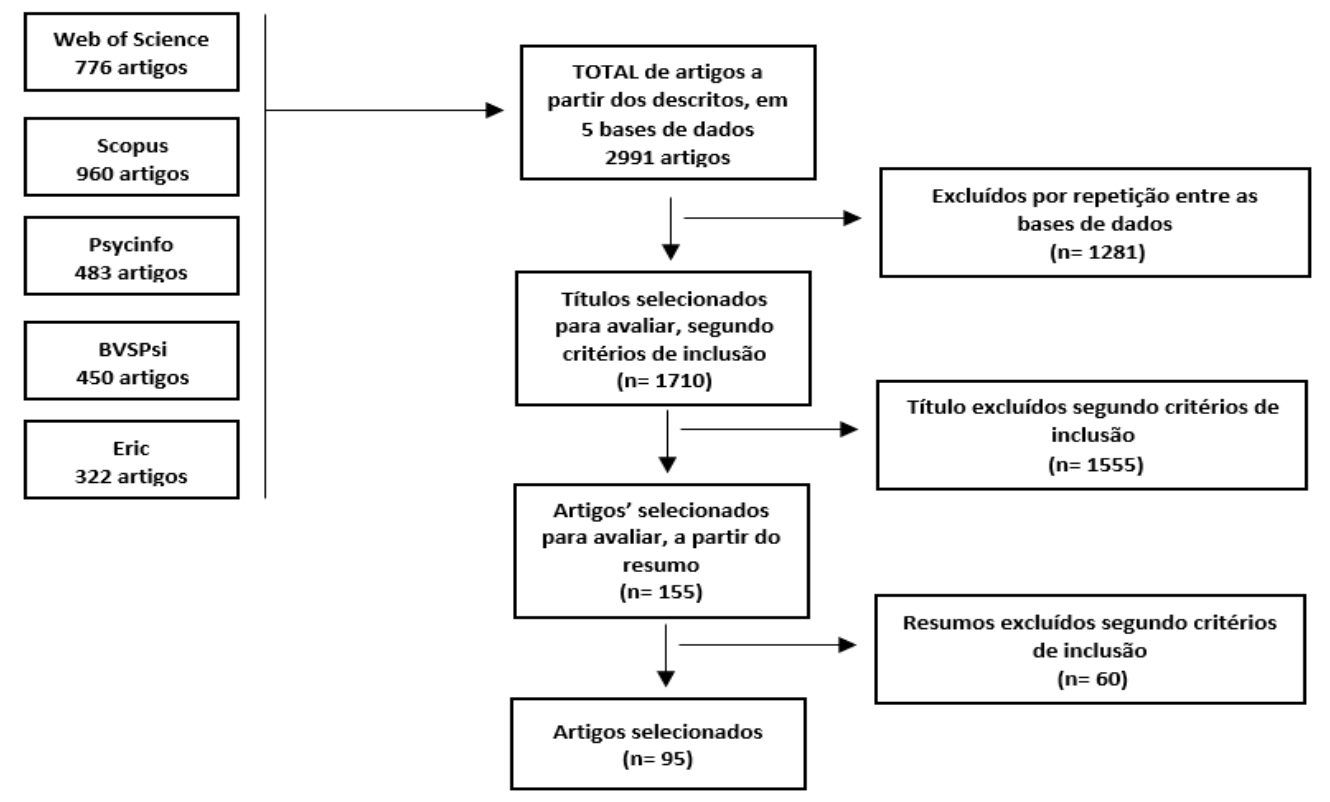

Fonte: elaborado pelos autores (2018). 
http://dx.doi.org/10.5902/1984686X41167

A maioria dos 95 artigos elegíveis foram publicados nos anos de 2014 e 2015, contabilizando 13 artigos em cada ano. Antes de 2014 e depois de 2015, a média de publicações elegíveis é entre 6 e 9 artigos em cada ano. Seguindo a proposta dos objetivos específicos, extraiu-se de cada artigo dados relacionados a diferentes campos de análise para a intervenção precoce abrangente, comportamental e/ou desenvolvimentista, com crianças com autismo.

\section{Áreas de desenvolvimento alvos das intervenções}

A pesquisa elegeu artigos que evidenciassem programas de intervenção precoce abrangentes, ou seja, cujas ações interventivas afetam o desenvolvimento infantil de modo amplo, envolvendo habilidades de comunicação, interação social, cognição, entre outros. Entretanto, dependendo do tipo de abordagem ou da teoria, ênfases podem ser diferentes. Por exemplo, no que se refere à linguagem, é tratada em termos de habilidades expressiva e receptiva (PETERS-SCHEFFER et al., 2011) ou, comunicação não-verbal (como contato visual) e desenvolvimento da fala, como é num programa do ESDM adaptado culturalmente, na China (XU et al., 2017).

No que se refere à interação social, os programas visam o desenvolvimento de habilidades de atenção conjunta, habilidades de brincar, imitação (RYBERG, 2015; WARREN et al., 2011; XU et al., 2017). Podem considerar como objetivo o desenvolvimento de comportamentos adaptativos, envolvendo flexibilidade comportamental, redução de comportamentos problemáticos (DEL SOL FORTEA; ESCANDELL; CASTRO, 2015). A motivação social da criança para interagir pode ser foco mais amplo e explícito de alguns programas de intervenção precoce para TEA (HERBRECHT et al., 2015).

Quanto ao campo cognitivo, estão envolvidas a inteligência não-verbal, a capacidade de simbolização, a inteligência verbal. Embora os programas não explicitem, em sua maioria, ênfase no desenvolvimento motor, essa área de desenvolvimento tende a ser controlada em termos de ganhos de habilidades (PAYNTER et al., 2015).

A capacidade de envolvimento interpessoal através de sincronia, ritmos e reciprocidade são alvos de programas como o ESDM, por exemplo, que visa diminuir a gravidade dos sintomas e acelerar o desenvolvimento cognitivo, socioemocional e de linguagem (RYBERG, 2015). Crianças que recebem intervenções de abordagem comportamental do tipo ABA tendem a ter como alvo o desenvolvimento cognitivo, linguagem e funcionamento adaptativo (EIKESETH, 2009). 
http://dx.doi.org/10.5902/1984686X41167

Embora o foco dos programas, em sua maioria, seja o desenvolvimento da criança, há os que visam, também, o desenvolvimento de habilidades dos pais, como habilidade para ser mais responsivo às interações da criança, o desenvolvimento de estratégias de regulação da emoção do filho (DABABNAH; PARISH, 2015) ou ainda a redução do estresse dos pais (D’ELIA et al., 2014). Tais aspectos podem ser tratados de forma explícita, como propósito do programa, ou conferidos como efeitos secundários em função da melhora dos filhos.

\section{Tempo médio de intervenção sistematizadas que as crianças devem receber}

Os programas de intervenção precoce, em sua maioria, partem do pressuposto de que as intervenções devem ser intensivas - o que representa cerca de 3 a 4 horas diárias (NARZISI et al., 2014). A intervenção comportamental intensiva precoce (EIBI), um tratamento baseado nos princípios da análise aplicada do comportamento, administrada por vários anos em uma intensidade de 20 a 40 horas por semana, é um dos tratamentos mais bem estabelecidos para o TEA (REICHOW et al., 2012). O caráter intensivo, no que se refere ao número mínimo de horas semanais, também é conferido ao Modelo Denver de Intervenção Precoce [(ESDM) - Early Star Denver Model] (VIVANTI et al., 2014; VIVANTI; DISSANAYAKE, 2016).

Dentre os estudos empíricos encontrados, há diversidade de propostas em termos de horas de intervenção, variando de 20-40 horas/semana (HOWARD et al., 2014; MOINÁR; ELDEVIK, 2016) a 2 a 5 sessões de 45 min por semana (DEL SOL FORTEA; ESCANDELL; CASTRO, 2015). Adaptações do programa Denver foram encontradas em escala de menor intensidade de intervenção, com 1 hora semanal de contato com o terapeuta, sendo recomendado aos pais que estendam as intervenções em casa (ROGERS et al., 2012). Num estudo realizado na China, adaptando o ESDM, as crianças do grupo controle receberam serviços de intervenção eclética por um mínimo de 2 horas por dia, 10 horas por semana, enquanto as crianças do grupo intervenção $(n=16)$ receberam a intervenção culturalmente adaptada 1 hora por dia, 5 hora por semana, mais 5 horas por semana dos mesmos serviços que o grupo controle, por um total de 8 semanas (XU et al., 2017).

O programa de intervenção FIAS [(FIAS) - Frühintervention bei autistischen Störungen] (praticado na Suíça e derivado do programa Mifne, de Israel) propõe que, durante 18 dias, terapeutas continuamente trabalhem com a criança com autismo durante $6 \mathrm{~h} /$ dia, envolvendo toda a família em uma creche (HERBRECHT et al., 2015). Sobre o 
http://dx.doi.org/10.5902/1984686X41167

programa PLAY, um estudo apresentou-se como de baixa intensidade, visto que os participantes receberam sessões mensais de intervenção entre pais e filhos durante 1 ano, durante os quais os pais aprenderam como usar as estratégias lógicas e interativas do modelo de intervenção Developmental, Individual-Differences, Relationship-based (DIR) (MAHONEY; SOLOMON, 2016). No programa Frankfurt, a frequência do tratamento é de 2 $\mathrm{h}$ / semana e reconhece-se que, embora haja ganhos cognitivos, eles são menores quando comparados com a maioria dos programas de alta intensidade (30-40 h / semana) (FREITAG et al., 2012).

Programas públicos, com intenção de maximizar a acessibilidade e custo-efetividade de seus serviços, podem implementar programas com intensidade e durações alternativas, a exemplo de um programa de um ano para pais, com sessões intensivas seguidas de uma hora por semana de treinamento individual, e de um programa de EIBI com menos intensidade do que o ideal, oferecida em ambientes de creches convencionais (RIVARD; TERROUX; MERCIER, 2014).

\section{Principais contextos e agentes das intervenções precoces}

Alguns formatos de programas de intervenção precoce trazem o contexto de intervenção ou o agente da intervenção ("quem intervém?") nos seus pressupostos, outros não: por exemplo, se a proposta é mais naturalista, o programa pode privilegiar o contexto doméstico e recomendar que os implementadores sejam os pais (SHANNON, 2015). Em outras situações, o contexto e os agentes de intervenção são condições anteriores à escolha da metodologia do programa, a exemplo de estudos que referem-se a intervenções em escolas ou centros de atendimento (COLOMBI et al., 2016; PAYNTER et al., 2015). Entre os estudos encontrados, tanto os contextos quanto os agentes são variáveis e, por vezes, combinam no programa mais de um contexto e mais de um tipo de interventor (HOWARD et al., 2014).

Quanto aos contextos, alguns estudos trazem propostas de intervenção em domicílio (D'ELIA et al., 2014; MOINÁR; ELDEVIK, 2016; PERERA et al., 2016; REED et al., 2013), serviços comunitários ou centros de atendimento (COLOMBI et al., 2016; EIKESETH, et al., 2012; PAYNTER et al., 2015; VIVANTI et al., 2014), escola (HERBRECHT et al., 2015) ou associando dois ou mais contextos, como escola e casa, por exemplo (D'ELIA et al., 2014; SOLOMON et al., 2014). 
http://dx.doi.org/10.5902/1984686X41167

Quando a intervenção tem a residência da criança como contexto-chave, tende a justificar-se em torno de criar e adotar o ensino estruturado em ambientes naturais e promover a generalização das habilidades da criança em diferentes contextos da sua vida (D'ELIA et al., 2014). No Programa PLAY, além dos serviços comunitários habituais, havia consulta domiciliar, com a intenção de melhorar a interação pai-criança, e os consultores do programa treinavam cuidadores para também melhorar a interação entre o cuidador e a criança (SOLOMON et al., 2014).

Em termos de contextos comunitários, o Programa Australiano de Intervenção Precoce Específico para Autismo, por exemplo, é um programa comunitário, cuja intervenções são realizadas em centros de intervenção especializados (PAYNTER et al., 2015). Há propostas intervencionistas que combinam diferentes ambientes, incluindo residências, centros de tratamento, ambientes comunitários e salas de aula regulares de pré-escola e ensino fundamental (HOWARD et al., 2014).

A razão pela qual um contexto é eleito para a intervenção nem sempre está relacionado ao que se compreende ser o melhor lugar para a criança desenvolver-se, mas muitas vezes, ao melhor lugar em determinadas circunstâncias ou condições. $O$ fato desta pesquisa ter como critério de inclusão estudos empíricos que tivessem, no mínimo, 10 participantes, pode ter produzido um viés na inclusão de artigos que predominassem intervenções comunitárias, visto que não houve inclusão de estudos com intervenções individuais em ambientes clínicos - o que possivelmente existe.

Programas de intervenção clínica individualizada e intensiva podem não estar prontamente disponíveis na comunidade, resultando em crianças com TEA que perdem a intervenção baseada em evidências durante anos críticos do desenvolvimento, que é a primeira infância. Por isso, há estudos que apresentam a intervenção precoce em formatos adaptados dos programas originais, como é o caso de vários estudos de EIBI ou ESDM comunitários (EAPEN; ČRNČEC; WALTER, 2013; MOINÁR; ELDEVIK, 2016; STAHMER, 2007).

O estudo de Eapen, Črnčec e Walter (2013) avaliou a eficácia do ESDM para crianças em idade pré-escolar com ASD usando uma intervenção predominantemente baseada em grupo em um ambiente comunitário de cuidados infantis. Outro estudo com EIBI domiciliar foi adaptado para um serviço baseado na comunidade com os pais funcionando como terapeutas primários (MOINÁR; ELDEVIK, 2016). Os programas comunitários precisam, em geral, adaptar programas "ideais" para o "mundo real" - o que levanta a necessidade de 
http://dx.doi.org/10.5902/1984686X41167

pesquisas futuras no sentido de desenvolver metodologias específicas para os programas que têm o contexto comunitário como contexto primário da intervenção (STAHMER, 2007), além de conferir a fidelidade de implementação quando as intervenções são realizadas em ambientes não controlados ou implementados por não-técnicos (pais, por exemplo).

Os pais têm sido envolvidos na implementação de estratégias de interação e gerenciamento de comportamento das crianças com TEA e os benefícios potenciais são o aumento de habilidades e a redução do estresse tanto para pais quanto para os filhos (MCCONACHIE; OONO, 2013; OONO; HONEY; MCCONACHIE, 2013). Os pais, contudo, precisam de treinamento e de apoio para lidar com as dificuldades: formação e apoio, somados a profissionais sensibilizados e tecnicamente capazes, tendem a produzir melhorias significativas no desenvolvimento de crianças com TEA (CANAL-BEDIA et al., 2014).

No FIAS, as intervenções ocorrem por terapeutas, que trabalham continuamente por 18 dias na creche que a criança frequenta, envolvendo a família (HERBRECHT et al., 2015). O programa Barnet Early Autism Model (BEAM) tem formato de intervenção que, embora seja no contexto doméstico, é uma equipe de multiprofissional (fonoaudiólogo, terapeuta ocupacional, psicólogo educacional) que realiza as intervenções em domicílio, com estreita colaboração com os pais da criança e outros membros da família (REED et al., 2013).

Numa intervenção com pré-escolares com autismo, baseado no Projeto de Autismo Jovem da Universidade da Califórnia em Los Angeles (UCLA YAP), os pais mediaram as intervenções com os filhos, sendo considerados terapeutas primários ou terapeutas ativos (MOINÁR; ELDEVIK, 2016). Em países com baixos recursos na saúde mental infantil, muitas crianças com TEA não têm recebido intervenção precoce, a não ser que os pais sejam treinados. Na Índia, por exemplo, no intuito de maximizar os recursos existentes, avaliou-se a viabilidade e eficácia de uma intervenção precoce intensiva, com múltiplos componentes, mediada por pais (KRISHNAN et al., 2016).

Além dos pais, programas de intervenção precoce também podem ser executados por educadores (D'ELIA et al., 2014; STRAIN; SCHWARTZ; BARTON, 2012; XU et al., 2017) ou profissionais peritos em autismo (DEL SOL FORTEA, ESCANDELL; CASTRO, 2015; JANSSON et al., 2016). Outras intervenções, ainda, têm sido implementadas por mais de um tipo de agente, como, por exemplo, no programa de intervenção precoce de Frankfurt (FFIP), em que tanto pais quanto educadores infantis são treinados para realizar as intervenções (FREITAG et al., 2012). 
http://dx.doi.org/10.5902/1984686X41167

Tão importante quanto considerar que pais e familiares são chave para o sucesso da intervenção precoce, também é considerar o risco aumentado de estresse, depressão, sentimentos de isolamento e falta de confiança para os pais/cuidadores de crianças com TEA - o que faz com que requeiram maior apoio informativo e emocional. Necessário, portanto, que haja pesquisas adicionais para desenvolver formas mais eficazes de fornecer apoio aos pais, diminuir o estresse e melhorar o senso de competência destes. Isso, sem dúvidas, melhorará a qualidade dos programas de intervenção (ESTES et al., 2013).

\section{Ingredientes ativos nos efeitos do tratamento}

Conhecer os ingredientes ativos num programa de intervenção é fundamental, para que se possa compreender qual elemento do programa contribui mais para efeitos de sua eficácia e, assim, possa-se dosar deliberadamente este ingrediente no planejamento e oferta da intervenção. Na maior parte dos estudos, contudo, os ingredientes ativos das intervenções não são ligados aos efeitos documentados, tendo os dados relativos à implementação das intervenção descritos frequentemente de forma resumida (CARON; BÉRUBÉ; PAQUET, 2017).

Os estudos considerados nesta revisão tinham como critério serem referentes a programas de intervenção precoce abrangentes, o que, em alguma medida, pode ter sido responsável por um viés no aspecto "ingrediente ativo". Em geral, os programas de intervenção abrangentes têm como característica, também, a recomendação de que sejam intensivos, no sentido de muitas horas de intervenção semanal. A intensidade dos tratamentos foi referida como ingrediente ativo em inúmeros estudos (CANAL-BEDIA et al., 2014; MAKRYGIANNI; REED, 2010; ROGERS et al., 2012; ROGERS; VISMARA, 2008; TONGE et al., 2014).

Em uma metanálise de 14 estudos sobre intervenções comportamentais para crianças com TEA, a intensidade foi responsável por um impacto maior no ganho de habilidades comportamentais intelectuais e adaptativas de crianças com TEA, mas não parece haver correlação com o progresso das crianças nas habilidades de linguagem (MAKRYGIANNI; REED, 2010). Já a revisão de Virués-Ortega (2010) com 22 estudos, também sobre intervenções comportamentais para crianças com TEA, não estabeleceu relações significativas entre comportamento adaptativo e intensidade do tratamento, mas corrobora que escores de linguagem não apresentam dependência da dose da intervenção. 
http://dx.doi.org/10.5902/1984686X41167

Tanto quanto a intensidade, a idade da criança com TEA ao iniciar o programa de intervenção também tem sido positivamente relacionado com o grau de melhoria no comportamento das crianças (CANAL-BEDIA et al., 2014; MAKRYGIANNI; REED, 2010; ROGERS et al., 2012). Num grupo de 83 crianças que receberam EIBI por um ano, os resultados mais significativos foram identificados entre aquelas que começaram o tratamento antes de completar dois anos de idade (MACDONALD et al., 2014).

Quanto mais cedo começar a intervenção e mais alto for o QI da criança nesse início do tratamento, maior a probabilidade de desfechos favoráveis após 12 e 24 meses de EIBI (SMITH; KLORMAN; MRUZEK, 2015). Crianças mais jovens podem alcançar ganhos superiores em desempenho cognitivo verbal, mas não haver diferença com os ganhos de colegas mais velhos no que se refere ao desempenho cognitivo não verbal ou comportamento adaptativo (VIVANTI; DISSANAYAKE, 2016). Uma revisão de literatura sobre EIBI concluiu, entretanto, que, mesmo com variabilidade considerável nos resultados das intervenções, algumas evidências apontam que não era a idade o ingrediente ativo nos progressos das crianças, mas o QI inicial (HOWLIN; MAGIATI; CHARMAN, 2009).

Há evidência científicas de que o envolvimento dos pais nas intervenções é também ingrediente ativo nas melhorias no desenvolvimento da criança. Abordagens que ajudam os pais a desenvolverem estratégias de interação e gerenciamento de comportamento são consideradas rota óbvia para a intervenção precoce no TEA (MAKRYGIANNI; REED, 2010; MCCONACHIE; OONO, 2013; TONGE et al., 2014). Um estudo randomizado envolvendo 112 crianças pré-escolares no Projeto PLAY [(PLAY) - Play and Language for Autistic Youngsters], uma intervenção precoce baseada em relações, demonstrou que o aumento na responsividade e afeto dos pais produziu efeitos no envolvimento social das crianças e nos transtornos do afeto social (MAHONEY; SOLOMON, 2016). O envolvimento dos pais nas intervenções com crianças com TEA tem sido enfatizado, incorporando oportunidades de aprendizado nas atividades cotidianas, pelo potencial de capitalizar os "momentos de aprendizado", facilitando a generalização da aprendizagem de habilidades para além do ambiente doméstico (CANAL-BEDIA et al., 2014; ZWAIGENBAUM et al., 2015).

Por fim, a duração de um programa de intervenção, habilidades de comportamento adaptativo das crianças no início do programa e multidisciplinaridade são elementos a serem considerados como determinantes os progressos da criança com TEA (MAKRYGIANNI; REED, 2010; TONGE et al., 2014). Em relação ao ESDM, concluiu-se que estudos adicionais são necessários para determinar os ingredientes ativos desse 
http://dx.doi.org/10.5902/1984686X41167

programa, além do nível de eficácia e se o ESDM é melhor que outras opções de tratamento (BARIL; HUMPHREYS, 2017).

\section{Indicadores de qualidade dos programas}

As pesquisas encontradas na revisão de literatura trouxeram, em sua maioria, indicadores explícitos de progresso no desenvolvimento das crianças que receberam intervenção. Em geral, por meio de instrumentos formais, diferenças entre pré e pós intervenção nos níveis de QI não verbal, linguagem expressiva e receptiva e comportamento adaptativo (PETERS-SCHEFFER et al., 2011).

O programa ESDM, mostrou-se promissor como uma prática baseada em evidências para crianças pequenas com TEA, embora não se possa afirmar que a extensão das melhorias observadas nas crianças é devido à intervenção desse modelo, por si só (BARIL; HUMPHREYS, 2017). Este programa mede evoluções no desenvolvimento da criança por meio dos indicadores de habilidades cognitivas, adaptativas e sociais, tendendo a haver maiores taxa de desenvolvimento e linguagem receptiva (COLOMBI et al., 2016; VIVANTI et al., 2014). Um estudo com objetivo de verificar atividade cerebral por meio de um eletroencefalograma (EEG) ao olhar para rostos, teve como indicador de eficácia do ESDM a maior ativação cortical das crianças com TEA ao visualizar rostos (próximo ao grupo de crianças com desenvolvimento típico), que é associada a um melhor comportamento social (DAWSON et al., 2012).

Tanto os estudos empíricos quanto as revisões de literatura sugerem que o EIBI é um tratamento eficaz. As intervenções de abordagem comportamentais, de longo prazo, levam a efeitos positivos médios a grandes, em favor do grupo de tratamento para todos os desfechos. Em termos de funcionamento intelectual, desenvolvimento da linguagem, comportamento adaptativo (aquisição de habilidades de vida diária e funcionamento social) das crianças com TEA. Resultados relacionados à linguagem (QI, linguagem receptiva e expressiva, comunicação) são superiores ao QI não verbal, funcionalidade social e habilidades de vida diária (ELDEVIK et al., 2009; HOWARD et al., 2014; REICHOW et al., 2012; VIRUÉS-ORTEGA, 2010). Os tamanhos de efeito para a linguagem receptiva tendem a ser mais altos do que a linguagem expressiva (REICHOW; WOLERY, 2009).

Num estudo de aplicação do EIBI, houve evidências de progressos no nível coletivo, cujo principal indicador de melhora foi O QI, quando comparado com grupo controle. Entretanto, no nível individual, houve considerável variabilidade na resposta ao tratamento, 
com algumas evidências de que o QI inicial (e não a idade) estava relacionado ao progresso. Esta revisão fornece evidências para a eficácia do EIBI para algumas crianças pré-escolares com TEA, mas não todas (HOWLIN; MAGIATI; CHARMAN, 2009).

Em uma revisão de literatura sobre intervenções mediadas pelos pais, encontrou-se evidências de eficácia com indicadores proximais na interação pais-criança e indicadores mais distais de compreensão da linguagem infantil e redução da gravidade do autismo (MCCONACHIE; OONO, 2013). O estudo de Solomon et al (2014) também indicou que houve mudanças na qualidade das interações entre pais e criança. Estudos que buscaram indicadores de qualidade dos programas de intervenção, por meio do efeito sobre os pais, encontraram resultados positivos em termos de satisfação e melhoria na implementação do tratamento pelos pais (BRADSHAW et al., 2015), bem como diminuição no estresse dos pais (D'ELIA et al., 2014; SOLOMON et al., 2014). Noutro estudo, dados qualitativos identificaram que os pais os pais se beneficiaram mais das estratégias de regulação da emoção infantil, das habilidades de comportamento infantil baseadas em brincadeiras, do gerenciamento do estresse dos pais, do apoio social e dos recursos visuais (DABABNAH; PARISH, 2015).

\section{Considerações finais}

As práticas interventivas com pessoas com autismo são sempre combinações de múltiplas estratégias e que requerem evidências científicas quanto à eficácia. Em se tratando de intervenção precoce, há a complexa tarefa de driblar variáveis intervenientes que também produzem mudanças no desenvolvimento da criança. Como saber, então, a quem se deve a responsabilidade por essas mudanças? É a abordagem de tratamento? A intensidade dos serviços? São as características particulares da criança e da família? A despeito da dificuldade de produzir respostas seguras a essas questões, é fundamental que se construam indicadores que sejam capazes de prever, em alguma medida, que tipo de tratamento ou intensidade de intervenção são os mais recomendados para cada criança, para que, em última análise, possa-se orientar melhor as intervenções e medir as mudanças atribuídas aos efeitos da intervenção.

A presente revisão de literatura evidenciou que programas de intervenção precoce para crianças com TEA têm assumido como alvo, principalmente, as seguintes áreas de desenvolvimento: habilidades sociais, de comunicação, cognição e habilidades adaptativas (COLOMBI et al., 2018; KRISHNAN et al., 2016). A compreensão sobre o impacto do 
http://dx.doi.org/10.5902/1984686X41167

autismo na saúde mental da família faz com que estudos se ocupem também de acompanhar estresse parental (REED et al., 2013), qualidade de vida dos pais (ROBERTS et al., 2011), aceitação dos pais, satisfação e melhoria na implementação do tratamento pelos pais (DABABNAH; PARISH, 2016). Há recomendação de que as intervenções possam ser intensivas (mais de 15 horas semanais), contudo, os estudos demonstram que, na prática, nem sempre é o possível, considerando-se os recursos econômicos da comunidade (KRISHNAN et al., 2016). Entretanto, mesmo quando a intervenção é de baixa intensidade, há ganhos relevantes em termos de ganho de habilidades ou redução de sintomas do autismo (D'ELIA et al., 2014; NAIR et al., 2014; ).

O contexto em que a intervenção precoce pode ocorrer varia desde ambientes clínicos, domésticos, escolares ou comunitários (centros de intervenção), predominando, nos estudos identificados, os dois primeiros contextos. A razão pela qual um programa ocorre num ou noutro contexto parece ter mais a ver com as possibilidades do que necessariamente da condição do programa. Os aplicadores das intervenções variam de profissionais especializados (em áreas de saúde ou educação) a pais treinados por profissionais.

Um programa de intervenção precoce tem maior resposta de efetividade quanto mais nova for a criança (CANAL-BEDIA et al., 2014; MAKRYGIANNI; REED, 2010; ROGERS et al., 2012) e, principalmente, quanto mais alto for o QI (HOWLIN; MAGIATI; CHARMAN, 2009; SMITH; KLORMAN; MRUZEK, 2015) e suas habilidades verbais. A intensidade também é uma condição que impacta em ganhos intelectuais e comportamentos adaptativos, mas não necessariamente em habilidades de linguagem (MAKRYGIANNI; REED, 2010). Para medir eficácia dos programas, normalmente se tem tomado como indicadores as evoluções das crianças nas áreas cognitivas, de linguagem, comportamento adaptativo e habilidades sociais.

A evidência de que tanto a idade mais jovem quanto a intensidade do tratamento afetam positivamente os progressos da criança têm implicações para a prática clínica, prestação de serviços e políticas públicas (CANAL et al., 2014; SMITH; KLORMAN; MRUZEK, 2015) . Não se tem registro de artigos publicados sobre os efeitos na vida adulta de tais tratamentos - uma pesquisa dessa natureza indicaria se a intervenção precoce resulta em uma dependência reduzida dos serviços de saúde na idade adulta (TONGE et al., 2014). Para que programas de intervenção sejam reconhecidos como práticas baseadas em evidências científicas, devem atender a seis critérios: a) foco no paciente; b) 
constituída de base científica; c) os resultados se baseiam na população alvo; d) a busca pela melhoria no padrão de qualidade; e) atendimento individualizado para cada paciente, e f) compatível com as políticas e os recursos do sistema (AKOBENG, 2005). Numa revisão de literatura futura, considera-se relevante: 1) uma avaliação de forças de evidências de cada estudo contemplado (verificando em quanto cada estudo atende aos seis critérios); 2) uma avaliação sobre o rigor metodológico dos estudos; 3) apresentação dos estudos com recursos visualmente mais informativos (em tabela, por exemplo) - neste caso, não realizado em função da extensa abrangência de dados coletados (95 artigos).

Esta revisão sistemática de literatura evidenciou as principais diretrizes para a implementação e programas de intervenção precoce, as quais destacam-se: 1) começar as intervenções tão logo se identifique risco do TEA, minimizando o intervalo entre diagnóstico e tratamento; 2) oferecer intervenção intensiva e sistemática (no mínimo, de 3 a 4 horas por dia); 3) intervenção com envolvimento da família; 4) acompanhar o desenvolvimento com avaliações periódicas, atualizando os objetivos de tratamento ao longo do processo. Outras diretrizes, ainda, podem ser incluídas, como: 5) incentivo à criança para a comunicação espontânea; 6) promover habilidades por meio de brincadeiras com os pares e 7) ao adquirir habilidades, incentivar a generalização e manutenção em contextos naturais (NARZISI et al., 2014).

\section{Referências}

AKOBENG, Anthony K. Principles of evidence based medicine. Archives of Disease in Childhood, v. 90, n. 8, p. 837-840, jul. 2005.

\section{ASSOCIAÇÃO AMERICANA DE PSIQUIATRIA - APA. (2014). DSM-5 - Manual} Diagnóstico e Estatístico de Transtornos Mentais. Porto Alegre: ArtMed.

BARIL, Erika M.; HUMPHREYS, Betsy P. An Evaluation of the Research Evidence on the Early Start Denver Model. Journal of Early Intervention, Durham, v. 39, n. 4, p. 321338, jul. 2017.

BEN-ITZCHAK, Ester; ZACHOR, Ditza A. Who benefits from early intervention in autism spectrum disorders? Research in Autism Spectrum Disorders. v. 5, n. 1, p. 345-350, 2011.

BOSA, Cleonice Alves. Autismo: intervenções psicoeducacionais. Revista Brasileira de Psiquiatria, São Paulo, v. 28(Supl I), p. S47-53, 2006. Dispnível em: http://www.scielo.br/pdf/rbp/v28s1/a07v28s1.pdf. Acesso em: 31 mar. 2020. 
BOSA, Cleonice Alves; ZANON, Regina Basso. Bases teóricas do desenvolvimento prélingüístico: implicações para o diagnóstico precoce do autismo. In Autismo - Vivências e Caminhos. São Paulo: Blucher, 2016.

BRADSHAW, Jessica. et al. Feasibility and Effectiveness of Very Early Intervention for Infants At-Risk for Autism Spectrum Disorder: A Systematic Review. Journal of Autism and Developmental Disorders, v. 45, n. 3, p. 778-794, mar., 2015.

CANAL-BEDIA, Ricardo. et al. Programas de cribado y atención temprana en niños con trastornos del espectro autista. Revista Neurología, v. 58(Supl 1), 123-127, fev., 2014. Disponível em < http://www.neurologia.com/pdf/Web/58S01/bIS01S123.pdf>. Acesso em: 30 jan. 2019

CANCINO, Miguel Higuera. Transtornos do desenvolvimento e da comunicação. Rio de Janeiro: WAK, 2013.

CARON, Valérie; BÉRUBÉ, Annie; PAQUET, Annie. Implementation evaluation of early intensive behavioral intervention programs for children with autism spectrum disorders: A systematic review of studies in the last decade. Evaluation and Program Planning, Canadá, v. 62, p. 1-8, jun., 2017.

COLOMBI, Costanza et al. Implementation of the Early Start Denver Model in an Italian community. Autism, Itália, v. 22, n. 2, p. 126-133, out., 2016.

CONTALDO, Annarita. et al. The social effect of "being imitated" in children with autism spectrum disorder. Frontiers in Psychology, v.7, mai., 2016.

D'ELIA, Lidia. et al. A Longitudinal Study of the Teacch Program in Different Settings: The Potential Benefits of Low Intensity Intervention in Preschool Children with Autism Spectrum Disorder. Journal of Autism and Developmental Disorders, v. 44, n. 3, p. 615-626, mar., 2014.

DABABNAH, Sarah; PARISH, Susan L. Feasibility of an empirically based program for parents of preschoolers with autism spectrum disorder. Autism, v. 20, n. 1, p. 85-95, fev., 2015.

DAWSON, Geraldine. et al. Early Behavioral Intervention Is Associated With Normalized Brain Activity in Young Children With Autism. Journal of the American Academy of Child \& Adolescent Psychiatry, EUA, v. 51 n.11, p. 1150-1159, nov., 2012.

DEL SOL FORTEA, María; ESCANDELL, Maria Olga; CASTRO, José Juan. Assessment of the efficacy of a program to improve the social communication and behavior of young children with autism spectrum disorders. Revista de Investigacion En Logopedia, Espanha, v.5, n. 2, p. 167-185, 2015. Disponível em: https://www.scopus.com/inward/record.uri?eid=2-s2.084948738941\&partnerID=40\&md5=dde631b1a38a0a4157c60dcec7d5081b. Acesso em: 31 mar. 2020

EAPEN, Valsamma; ČRNČEC, Rudi; WALTER, Amelia. Clinical outcomes of an early 
http://dx.doi.org/10.5902/1984686X41167

intervention program for preschool children with Autism Spectrum Disorder in a community group setting. BMC Pediatrics, v. 13 n. 3, p. 1-9, jan., 2013.

EIKESETH, Svein. Outcome of comprehensive psycho-educational interventions for young children with autism. Research in Developmental Disabilities, v. 30 n. 1, p. 158-178. fev., 2009.

EIKESETH, Svein. et al. Outcome for children with autism receiving early and intensive behavioral intervention in mainstream preschool and kindergarten settings. Research in Autism Spectrum Disorders, v. 6, n. 2, p. 829-835, jun., 2012.

ELDEVIK, Sigmund. et al. Meta-Analysis of Early Intensive Behavioral Intervention for Children With Autism. Journal of Clinical Child \& Adolescent Psychology, v., 38, n. 3, p. 439-450, 2009.

ESTES, Annette. et al. The impact of parent-delivered intervention on parents of very young children with autism. Journal of Autism and Developmental Disorders, v. 44, $n$. 2, jul., 2013.

FREITAG, Christine M. et al. The Frankfurt early intervention early intervention program FFIP for preschool aged children with autism spectrum disorder: a pilot study. Journal of Neural Transmission, v. 119, n. 9, 1011-1021, 2012.

HERBRECHT, Evelyn. et al. Become Related: FIAS, an Intensive Early Intervention for Young Children with Autism Spectrum Disorders. Psychopathology, Suíça, v. 48, n. 3, p. 162-172, 2015.

HOWARD, Jane S. et al. A comparison of intensive behavior analytic and eclectic treatments for young children with autism. Research in Developmental Disabilities, $v$. 26, n. 4, p. 359-383, 2005.

HOWARD, Jane S. et al. Comparison of behavior analytic and eclectic early interventions for young children with autism after three years. Research in Developmental

Disabilities, EUA, v. 35, n. 12, p. 3326-3344, dez., 2014.

HOWLIN, Patricia; MAGIATI, Iliana; CHARMAN, Tony. Systematic Review of Early Intensive Behavioral Interventions for Children With Autism. American Journal on Intellectual and Developmental Disabilities, Londres, v. 114, n. 1, p. 23-41, jan., 2009.

JANSSON, Birgitta Spjut. et al. Children who screen positive for autism at 2.5 years and receive early intervention: A prospective naturalistic 2-year outcome study.

Neuropsychiatric Disease and Treatment, v. 12, p. 2255-2263, nov., 2016.

KRISHNAN, Raman. et al. The effectiveness of an intensive, parent mediated, multicomponent, early intervention for children with autism. Journal of Indian Association for Child \& Adolescent Mental Health, v. 12, n. 3, p. 230-252, 2016.

LAMPREIA, Carolina. A perspectiva desenvolvimentista para a intervenção precoce no autismo. Estudos de Psicologia, Campinas, v. 24, n. 1, p. 105-114, 2007. 
MACDONALD, Rebecca. et al. Assessing progress and outcome of early intensive behavioral intervention for toddlers with autism. Research in Developmental Disabilities, v. 35, n. 12, p. 3632-3644, dez., 2014.

MAHONEY, Gerald; SOLOMON, Richard. Mechanism of Developmental Change in the PLAY Project Home Consultation Program: Evidence from a Randomized Control Trial. Journal of Autism and Developmental Disorders, v. 46, n. 5, p. 1860-1871, jan., 2016.

MAKRYGIANNI, Maria K.; REED, Phill. A meta-analytic review of the effectiveness of behavioural early intervention programs for children with Autistic Spectrum Disorders.

Research in Autism Spectrum Disorders, v. 4, n. 4, p. 577-593, out., 2010.

MARQUES, Daniela Fernandes; BOSA, Cleonice Alves. Protocolo de Avaliação de Crianças com Autismo: Evidências de Validade de Critério. Psicologia: Teoria e Pesquisa, Brasília, v. 31, n. 1, p. 43-51, mar., 2015.

MARTOS-PÉREZ, Juan; LLORENTE-COMÍ, María. Tratamiento de los trastornos del espectro autista: unión entre la comprensión y la práctica en la evidencia. Revista de Neurología, v. 57(Supl 1), s185-s191, 2013. Disponível em:

http://centroalgoritmus.com/pdfs/articulos-autismo-general/union-entre-comprensiontratamientos.pdf. Acesso em: 31 mar. 2020.

MCCONACHIE, Helen; OONO, Inalegwo. Cochrane in context: Parent-mediated early intervention for young children with autism spectrum disorders (ASD). Evidence-Based Child Health: A Cochrane Review Journal, v. 8, n. 6, p. 2483-2485, 2013.

MOINÁR, Claire; ELDEVIK, Sigmund. Behavioral intervention for preschool children with autism - outcome of parent-based Intervention. Z Kinder Jugendpsychiatr Psychother, v. 45, n. 3, p. 181-191, set., 2016.

NAIR, M. K. C. et al. CDC Kerala 9: Effectiveness of Low Intensity Home Based Early Intervention for Autism Spectrum Disorder in India. Indian Journal of Pediatrics, v. 81, n. 2, p. 115-119, 2014.

NAIR, M. K. C. et al. CDC Kerala 8: Effectiveness of a Clinic Based, Low Intensity, Early Intervention for Children with Autism Spectrum Disorder in India: A Naturalistic Observational Study. Indian Journal of Pediatrics, v. 81. n. 2, p. 110-114, 2014.

NARZISI, Antonio. et al. Non-Pharmacological Treatments in Autism Spectrum Disorders: An Overview on Early Interventions for Pre-Schoolers. Current Clinical Pharmacology, v. 9, n. 1, p. 17-26, 2014.

OONO, Inalegwu P.; HONEY, Emma J.; MCCONACHIE, Hellen. Parent-mediated early intervention for young children with autism spectrum disorders (ASD). Evidence-Based Child Health, v. 8 n. 6, p. 2380-2479, nov., 2013.

OZONOFF, Sally. et al. A prospective study of the emergence of early behavioral signs of autism. Journal of the American Academy of Child and Adolescent Psychiatry, EUA, v. 49, n. 3, p. 256-66.e1-2, mar., 2010. 
PAYNTER, Jessica M. et al. Brief Report: An Evaluation of an Australian Autism-Specific, Early Intervention Programme. International Journal of Special Education, v. 30, n. 2, p. 13-19, 2015.

PERERA, Hemamali. et al. Outcome of home-based early intervention for autism in Sri Lanka: Follow-up of a cohort and comparison with a nonintervention group. BioMed Research International, p. 2-7, jun., 2016.

PETERS-SCHEFFER, Nienke. et al. A meta-analytic study on the effectiveness of comprehensive ABA-based early intervention programs for children with Autism Spectrum Disorders. Research in Autism Spectrum Disorders, v. 5, n. 1, p. 60-69, jan., 2011.

REED, Phil. et al. Evaluation of the Barnet Early Autism Model (BEAM) teaching intervention programme in a 'real world' setting. Research in Autism Spectrum Disorders, UK, v. 7, n. 6, p. 631-638, jun., 2013.

REICHOW, Brian. et al. Early intensive behavioral intervention (EIBI) for young children with autism spectrum disorders (ASD) (Review) Early intensive behavioral intervention (EIBI) for young children with autism spectrum disorders (ASD). Wiley Publishers, EUA, n. 10, p. 2-4, out., 2012.

REICHOW, Brian; WOLERY, Mark. Comprehensive Synthesis of Early Intensive Behavioral Interventions for Young Children with Autism Based on the UCLA Young Autism Project Model. Journal of Autism and Developmental Disorders, v. 39, n. 1, p. 23-41, jan., 2009.

RIVARD, Mélina; TERROUX, Amélie; MERCIER, Céline. Effectiveness of early behavioral intervention in public and mainstream settings: The case of preschool-age children with autism spectrum disorders. Research in Autism Spectrum Disorders, v. 8, n. 9, p. 1031-1043, set., 2014.

ROBERTS, Jacqueline. et al. A randomised controlled trial of two early intervention programs for young children with autism: Centre-based with parent program and homebased. Research in Autism Spectrum Disorders, v. 5, n. 4, p. 1553-1566, out., 2011.

ROGERS, Sally J.; VISMARA, Laurie. A. Evidence-Based Comprehensive Treatments for Early Autism. Journal of Clinical Child and Adolescent Psychology, v. 37, n. 1, p. 838, jan., 2008.

ROGERS, Sally J. et al. Effects of a Brief Early Start Denver Model (ESDM)-Based Parent Intervention on Toddlers at Risk for Autism Spectrum Disorders: A Randomized Controlled Trial. Journal of the American Academy of Child \& Adolescent Psychiatry, v. 51, n. 10, p. 1052-1065, out., 2012.

ROGERS, Sally J.; DAWSON, Geraldine. Intervenção Precoce em Crianças com Autismo: Modelo Denver para a promoção da linguagem, da aprendizagem e da socialização. Lisboa: Lidel, 2014.

RYBERG, Kayce. H. Evidence for the Implementation of the Early Start Denver Model for 
http://dx.doi.org/10.5902/1984686X41167

Young Children With Autism Spectrum Disorder. Journal of the American Psychiatric Nurses Association, v. 21, n. 5, p. 327-337, out., 2015.

SÁNCHEZ-RAYA, Araceli. et al. La atención temprana en los trastornos del espectro autista (TEA). Psicologia Educativa, Espanha, v. 21, n. 1, p. 55-63, jun. 2015.

SALVADÓ-SALVADÓ, Berta. et al. Modelos de intervención global en personas con trastorno del espectro autista. Revista de Neurología, v. 54, n. 1, p. 63-71, 2012. Disponível em <http://dugidoc.udg.edu/bitstream/handle/10256/12558/025355.pdf?sequence=1>. Acesso em 31 mar. 2020.

SHANNON, Robin Adair. A Clinical Translation of the Article Titled "Evidence for the Implementation of the Early Start Denver Model for Young Children With Autism Spectrum Disorder." Journal of the American Psychiatric Nurses Association, EUA, v. 21, n. 5, p. 338-342, out., 2015.

SMITH, Tristram; KLORMAN, Rafael; MRUZEK, Daniel W. Predicting Outcome of Community-Based Early Intensive Behavioral Intervention for Children with Autism. Journal of Abnormal Child Psychology, v. 43, n. 7, p. 1271-1282, out., 2015.

SOLOMON, Richard. et al. PLAY Project Home Consultation Intervention Program for Young Children With Autism Spectrum Disorders: A Randomized Controlled Trial. Journal of Developmental and Behavioral Pediatrics, v. 35, n. 8, p. 475-485, out., 2014.

STAHMER, Aubyn. C. The basic structure of community early intervention programs for children with autism: Provider descriptions. Journal of Autism and Developmental Disorders, v. 37, n. 7, p. 1344-1354, ago., 2007.

STRAIN, Phillip. S.; SCHWARTZ, Ilene S.; BARTON, Erin. Providing Interventions for Young Children With Autism Spectrum Disorders. Journal of Early Intervention, v. 33, n. 4, p. 321-332, jan., 2012.

TOMASELLO, Michael. Origens culturais da aquisição do conhecimento humano. São Paulo: Martins Fontes, 2003.

TOMASELLO, Michael. Origins of human communication. Massachussetts: The MIT Press, 2008.

TOMASELLO, Michael. et al. Understanding and sharing intentions: the origins of cultural cognition. Behavioral and Brain Sciences, v. 8, n. 5, p. 675-735, 2005.

TONGE, Bruce. et al. A review of evidence-based early intervention for behavioural problems in children with autism spectrum disorder. Current Opinion in Psychiatry, v. 27, n. 2, p. 158-165, jan., 2014.

VIRUÉS-ORTEGA, Javier. Applied behavior analytic intervention for autism in early childhood: Meta-analysis, meta-regression and dose-response meta-analysis of multiple outcomes. Clinical Psychology Review, Espanha, v. 30, n. 4, p. 387-399, jun., 2010. 
VIVANTI, Giacomo; DISSANAYAKE, Cheryl. Outcome for Children Receiving the Early Start Denver Model Before and After 48 Months. Journal of Autism and Developmental Disorders, v. 46, n. 7, p. 2441-2449, jul., 2016.

VIVANTI, Giacomo. et al. Effectiveness and Feasibility of the Early Start Denver Model Implemented in a Group-Based Community Childcare Setting. Journal of Autism and Developmental Disorders, EUA, v. 44, n. 12, p. 3140-3153, dez., 2014.

WARREN, Zachary. et al. A Systematic Review of Early Intensive Intervention for Autism Spectrum Disorders. Pediatrics, v. 127, n. 5, p. e1303-e1311, mai., 2011.

XU, Yun. A Pilot Study of a Culturally Adapted Early Intervention for Young Children With Autism Spectrum Disorders in China. Journal of Early Intervention, China, v. 40, n. 1, p. 52-68, dez., 2017.

ZANON, Regina Basso; BACKES, Bárbara; BOSA, Cleonice Alves. Diferenças conceituais entre resposta e iniciativa de atenção compartilhada. Revista Psicologia: Teoria e Prática, São Paulo, v. 17, n. 2, p. 78-90, ago., 2015.

ZANON, Regina Basso; BACKES, Bárbara; BOSA, Cleonice Alves. Diagnóstico do autismo: relação entre fatores contextuais, familiares e da criança. Revista Psicologia: Teoria e Prática, São Paulo, v. 19, n. 1, p. 152-163, abr., 2017.

ZWAIGENBAUM, Lonnie. et al. Early Intervention for Children With Autism Spectrum Disorder Under 3 Years of Age: Recommendations for Practice and Research. Pediatrics, v. 136(Supplement), p. S60-S81, out., 2015.

\section{Notas}

1 O Banco de dados Eric não disponibilizara artigos anteriores a 2009.

\section{Correspondência}

Ana Carolina Wolff Mota - Mediação Serviços em Psicologia, Psicologia clínica, Rua Marcílio Dias, 384, Santo Antonio, Joinville, Santa Catarina - Brasil.

CEP: 89202-010

\section{(i) (8)}

EY ${ }_{\mathrm{NC}}$ This work is licensed under a Creative Commons Attribution-NonCommercial 4.0 International (CC BY-NC 4.0) 